Relations industrielles

Industrial Relations

\title{
The Worker and the Job, The American Assembly, by Jerome M. Rosow, Ed., N.J. Englewood Cliffs, Prentice Hall, 1974, 208 pp.
}

\section{Maurice Lemelin}

Volume 32, numéro 2, 1977

URI : https://id.erudit.org/iderudit/028796ar

DOI : https://doi.org/10.7202/028796ar

Aller au sommaire du numéro

Éditeur(s)

Département des relations industrielles de l'Université Laval

ISSN

0034-379X (imprimé)

1703-8138 (numérique)

Découvrir la revue

Citer ce compte rendu

Lemelin, M. (1977). Compte rendu de [The Worker and the Job, The American Assembly, by Jerome M. Rosow, Ed., N.J. Englewood Cliffs, Prentice Hall, 1974, 208 pp.] Relations industrielles / Industrial Relations, 32(2), 288-289.

https://doi.org/10.7202/028796ar

Tous droits réservés @ C Département des relations industrielles de l'Universite Laval, 1977
Ce document est protégé par la loi sur le droit d'auteur. L'utilisation des services d'Érudit (y compris la reproduction) est assujettie à sa politique d'utilisation que vous pouvez consulter en ligne.

https://apropos.erudit.org/fr/usagers/politique-dutilisation/ 
dustry. Those who are squeamish about quantitative methods should be forewarned that this is not an easy book to read.

\section{Joseph B. ROSE}

School of Administration

University of New Brunswick

The Development of an African Working Class, by R. Sandbrook and R. Cohen, Toronto, University of Toronto Press, 1976, $330 \mathrm{pp}$.

C'est un colloque tenu à l'Université de Toronto en avril 1973 qui fut l'occasion de la présentation des textes réunis sous ce titre. Les auteurs se sont interrogés sur l'émergence d'une classe ouvrière africaine et sur les voies d'un changement de la condition des travailleurs sur le continent africain: ce changement est-il mieux garanti par la réforme ou par la révolution? Question ancienne, souvent reformulée depuis deux siècles par ceux qui ont souhaité des changements sociaux, qui ici est posée dans un contexte géographique et social différent.

La presque totalité de ceux qui ont contribué à ce recueil postulent que les sociétés africaines doivent changer et que ce changement doit se faire dans le sens de l'égalitarisme, donc de l'éradication du colonialisme et du racisme. Là où l'accord est plus difficile, c'est sur les moyens d'y parvenir: changement progressif, changement radical, modèle capitaliste, modèle socialiste, etc...?

Le lecteur ne trouvera pas de réponses définitives dans cet ouvrage; il pourra cependant documenter ses questions en prenant connaissance d'analyses d'historiens et de sociologues sur les premiers développements d'une conscience de classe ouvrière en Afrique, sur les organisations de travailleurs ainsi que sur les pratiques ouvrières contemporaines. Les éditeurs présentent des études de cas menées dans divers pays, en particulier au Nigéria, au Kénya, au Sénégal, au Ghana et en Tanzanie, dont l'expérience socialiste fait l'objet de deux articles. Une bibliographie de titres choisis accompagne la douzaine d'articles de ce recueil, à l'intention de ceux qui voudraient en savoir plus sur l'organisation ouvrière en Afrique.

Gilles DUSSAULT

Université Laval
The Worker and the Job, The American Assembly, by Jerome M. Rosow, Ed., N. J. Englewood Cliffs, Prentice Hall, $1974,208 \mathrm{pp}$.

Dans ce volume, sept (7) experts se penchent sur le travailleur américain et le monde du travail. Le fruit de leurs réflexions, recherches et expériences est rapporté en trois (3) parties qui ont comme titre: «Overview», «Contemporary Issues» et «New Horizons».

Tel que nous l'indique son titre, la première partie donne un aperçu général de la situation tant sur le plan sociologique qu'économique. Elle est divisée en deux chapitres intitulés "The Meaning of Work " et «The Changing American Economy».

Le premier chapitre, écrit par Daniel Yankelovich, est une réflexion sur la signification du travail. Après avoir tracé les origines de l' "American Work Ethic", l'auteur s'attarde à donner ses composantes essentielles dans les années 60. Il examine ensuite les principaux changements susceptibles de modifier la façon de concevoir le travail, à savoir la nouvelle définition du succès, la réduction de la peur de l'insécurité économique, la nouvelle division du travail entre les sexes, l'augmentation des attentes et la désillusion à l'égard du culte de l'efficacité. Toutefois selon Yankelovich, ces transformations n'atteignent pas tous les travailleurs également. Trois (3) groupes sont en fait plus particulièrement touchés: les jeunes instruits, les jeunes non instruits et les femmes. La réaction de ces groupes est un bon indicateur de ce que sera l'attitude future vis-à-vis le travail.

Dans le deuxième chapitre, Eli Genzberg nous fournit les principales données statistiques au sujet de la main-d'œuvre et des emplois. Comme il le souligne luimême, son but n'est pas de prouver ou de rejeter une théorie mais simplement de donner un cadre de référence, soit la comparaison et l'évolution entre les années 20 et 70. Ginzberg s'aventure toutefois à faire quelques projections dans le futur.

La deuxième partie du volume "Contemporary Issues» débute par un article de George Strauss sur les travailleurs, leurs attitudes et leurs adaptations. Il attire notre attention sur les questions suivantes: Jusqu'où les travailleurs sont-il insatisfaits? Cette insatisfaction est-elle essentiellement due au travail répétitif et monotone ou a-t-elle aussi pour cause les salaires, la supervision ou l'absence de mobilité? Estce que cette insatisfaction augmente? 
Heureusement ou malheureusement ce chapitre n'apporte pas de réponses définitives et concluantes à ces questions. Strauss laisse au lecteur le soin de tirer sa propre conclusion ou plutôt de poser ses propres hypothèses. En effet, il souligne d'abord qu'aucune preuve évidente ne semble soutenir l'énoncé que les travailleurs sont de plus en plus insatisfaits. Par ailleurs, il n'y a pas non plus de preuves contraires. Selon l'auteur, il est peut-être faux de prétendre que tous les travailleurs ressentent les mêmes besoins à l'égard du travail. Les théories avancées, par exemple par Maslow, McClelland ou autres, ne peuvent peut-être pas s'appliquer à tous les travailleurs. Car il est possible que pour certains, le travail ne soit qu'instrumental. De même il est probable que les travailleurs s'ajustent à leur travail.

Le quatrième chapitre traite du rôle des syndicats. Selon son auteur, Agis Salpukas, l'humanisation du travail présente un défi aux syndicats qui entraînera une redéfinition de leur rôle. Mais il constate que plusieurs chefs ouvriers demeurent sceptiques à l'égard du mouvement vers l'humanisation du travail.

Dans ce chapitre, Salpukas fait d'abord un bref rappel historique du mouvement ouvrier et des luttes syndicales. Ces luttes ont essentiellement porté sur la reconnaissance syndicale et les conditions physiques et matérielles reliées au travail. Cependant note l'auteur, les travailleurs ont maintenant d'autres attentes. Or, il constate que les syndiqués font peu appel à leur agent négociateur pour répondre à ces attentes. De plus, certaines exigences, telles la nature même de la négociation collective, posent certaines contraintes aux syndicats. Toutefois, et il termine sur cette note optimiste, il constate un désir croissant de la part de certains chefs ouvriers de travailler à l'humanisation du travail.

Le dernier chapitre de cette partie porte sur les effets économiques de l'insatisfaction au travail. Le problème auquel s'adresse Peter Henle est de savoir dans quelle mesure le changement dans les attitudes vis-à-vis le travail se reflète dans l'économie. Pour répondre à cette question, il divise le chapitre en quatre (4) sections qui sont l'apport de la main-d'œuvre, le rendement au travail, la productivité et les relations du travail.

Pour Henle, le changement dans les attitudes peut avoir un impact sur l'apport des travailleurs de quatre (4) façons: moins d'individus peuvent désirer travailler ou peuvent désirer travailler moins d'heures et ceux qui travaillent peuvent être plus enclins à s'absenter ou à quitter leur emploi. De la même façon l'insatisfaction au travail peut se refléter sur le rendement au travail et la productivité dans son ensemble. Le climat des relations du travail, à savoir par exemple le nombre de grèves ou de griefs, peut également indiquer un malaise profond. Mais l'auteur constate qu'en fonction de ces critères, il ne semble pas y avoir plus d'insatisfaction au travail. Du moins, s'il y a insatisfaction, elle ne se reflète pas dans l'économie.

La troisième partie du volume nous ouvre la porte sur de nouveaux horizons. Cette partie débute par l'écrit de Richard E. Walton intitulé «Innovative Restructuring of Work». Dans ce chapitre, l'auteur relate douze (12) cas de restructuration des tâches qui ont eu lieu dans onze (11) compagnies localisées dans cinq (5) pays. Walton dégage de cette revue sept (7) conditions facilitant la mise en place de projets semblables. À l'inverse, l'auteur fait également part des obstacles à l'élaboration, la persistance et la diffusion de changements organisationnels.

Enfin, Sam Zagoria poursuit en analysant les éléments incitant au statu quo. Il porte son analyse sur trois (3) groupes, soit les travailleurs, les syndicats et les employeurs. Il regarde ensuite les développements que connaissent certains pays. Il termine par une énumération d'actions que le gouvernement, les employeurs et les syndicats devront prendre pour s'adapter à la situation présente et faire face aux changements.

Somme toute, ce livre fait par des américains s'adresse d'abord aux américains. Quoique le problème de l'aliénation au travail ne soit pas particulier aux ÉtatsUnis, les études ou réflexions rapportées dans ce volume sont essentiellement orientées vers ce pays. Ainsi, les chapitres traitant par exemple de l'économie ou des effets économiques présentent un intérêt beaucoup moins grand pour nous. Il en est de même des chapitres ou sections discutant de l' "American Work Ethic». Par ailleurs, mises à part ces remarques qui sont, d'une certaine façon, majeures, ce volume apporte certains éléments de réflexion.

\section{Maurice LEMELIN}

École des Hautes Études Commerciales 